\title{
Distribuição dos óbitos por câncer de colo do útero no Brasil
}

\section{Distribution of deaths from cervical cancer in Brazil}

\author{
Thaís Aurora Alves Fonseca ${ }^{1}$ (i), Daniela Tamires Alves da Silva ${ }^{2}$ (), Maria Tatiane Alves da Silva ${ }^{3}$ (])
}

1. Graduanda no curso de Biomedicina na Universidade da Vitória de Santo Antão (UNIVISA); Vitória de Santo Antão, PE, Brasil. 2. Graduanda no curso de Fisioterapia na Universidade FACOL (UNIFACOL), Vitória de Santo Antão, PE, Brasil. 3. Mestranda no Programa de Pós-graduação em Saúde Pública do Instituto Aggeu Magalhães/Fundação Oswaldo Cruz (IAM/FIOCRUZ), Recife, PE, Brasil

\section{Resumo}

Objetivo: descrever a distribuição dos óbitos por câncer de colo do útero no Brasil. Métodos: trata-se de um estudo ecológico e descritivo, com uma abordagem quantitativa. Foram utilizados dados secundários, disponibilizados no Sistema de Informações sobre Mortalidade e no Instituto Brasileiro de Geografia e Estatística, dos anos de 2010 a 2019. As variáveis utilizadas no estudo foram ano, localização geográfica, raça/cor, escolaridade e faixa etária. Os dados foram apresentados em tabelas, gráfico e mapa, por meio de frequências absolutas e relativas, sendo utilizados os softwares Excel e o SIG QGIS 3.16. Resultados: o Brasil apresentou uma tendência crescente para o número de óbitos e Taxa de Mortalidade por Câncer de Colo do Útero. A prevalência foi maior na população negra (53,66\%); na faixa etária de 40 a 59 anos (42,00\%) e 60 anos ou mais (42,47\%); e com apenas 1 a 3 anos de escolaridade $(23,69 \%)$. Além disso, em relação à distribuição geográfica das taxas de mortalidade, nota-se que não é uniforme no território nacional. No geral, destacamse os estados das regiões Norte e Nordeste, em especial, o estado do Amazonas. Conclusões: a análise da distribuição dos óbitos por câncer de colo do útero é uma estratégia para identificação de populações vulneráveis, contribuindo para o direcionamento das políticas públicas voltadas para a prevenção da neoplasia.

Palavras-chave: Neoplasias do Colo do Útero; Mortalidade; Saúde da Mulher; Saúde Pública.

\begin{abstract}
Objective: to describe the distribution of deaths from cervical cancer in Brazil. Methods: This is an ecological and descriptive study, with a quantitative approach. Secondary data were used, made available in the Mortality Information System and the Brazilian Institute of Geography and Statistics, from 2010 to 2019. The variables used in the study were year, geographic location, race/color, education, and age group. The data were presented in tables, graphs and maps, using absolute and relative frequencies, and the software Excel and the SIG QGIS 3.16 were used. Results: Brazil showed an increasing trend for the number of deaths and mortality rate from cervical cancer. The prevalence was higher in the black population (53,66\%); in the age group of 40 to 59 years old $(42,00 \%)$ and 60 years or more $(42,47 \%)$; and with only 1 to 3 years of schooling $(23,69 \%)$. Furthermore, regarding the geographic distribution of mortality rates, it is noted that it is not uniform across the national territory. In general, the states of the North and Northeast regions stand out, especially the state of Amazonas. Conclusions: The analysis of the distribution of deaths from cervical cancer is a strategy for identifying vulnerable populations, contributing to the direction of public policies aimed at preventing cancer.
\end{abstract}

Keywords: Uterine Cervical Neoplasms; Mortality; Women's Health; Public Health.

\section{INTRODUÇÃO}

O câncer de colo do útero é um tumor maligno situado no tecido epitelial no colo do útero, proveniente das multiplicações anormais e descontroladas nas células da superfície cervical, desenvolvendo-se de forma gradativa e lenta ${ }^{1}$. A neoplasia é o terceiro carcinoma mais prevalente na população feminina, com altos índices de mortalidade. No ano de 2016, foram estimados 16.340 casos novos no Brasil, indicando um risco de 15,85 casos confirmados a cada 100 mil mulheres, sendo um dos principais problemas de saúde na população feminina ${ }^{2,3}$.

Em relação aos fatores de risco, a quantidade de parceiros, a falta da higienização íntima, o tabagismo e as situações socioeconômicas são alguns que se destacam ${ }^{1}$. No entanto, o principal fator que ocasiona a neoplasia é a constância infecção pelo Papilomavírus Humano (HPV), em que existem mais de 200 genótipos, podendo ser dividido em baixo e alto grau. Os genótipos de baixo grau não são considerados oncogêncicos; os de alto grau, sim, sendo os genes do tipo 16 e 18 responsáveis por $70 \%$ dos casos de câncer de colo do útero 4 .

As formas de prevenção do câncer de colo do útero ocorrem, especialmente, pela vacinação contra o HPV; instrução com relação ao uso do preservativo e redução do tabagismo. Além disso, por meio do exame citopatológico, é possível detectar o câncer precocemente. 0 exame, também conhecido como Papanicolau, consiste no ato de coletar amostra do fundo do saco vaginal posterior e do ectocérvice, sendo responsável por identificar células pré-cancerosas e, dessa forma, tratar antes 
de se tornar uma neoplasia5,6.

No âmbito do Sistema Único de Saúde (SUS), o controle da neoplasia é realizado por meio de ações e serviços de saúde organizados de forma integral, sendo ofertadas ações desde a promoção e prevenção até o tratamento e reabilitação. No entanto, o Brasil é marcado, historicamente, por desigualdades sociais, sendo refletidas na qualidade de vida e saúde da população. A organização dos serviços de saúde é realizada por meio do contexto local, podendo ser identificada como um fator facilitador ou limitador do acesso da população aos serviços ${ }^{7}$.

O controle do câncer de colo do útero está condicionado às desigualdades sociais, econômicas e culturais. Aspectos como a renda e a escolaridade apresentam relação direta com a ocorrência de óbitos pela neoplasia e o acesso a ações e serviços voltados para a prevenção. Além disso, a mortalidade pode ser correlacionada com o acesso a informações à saúde ${ }^{7,8}$. Dessa forma, o objetivo do estudo foi analisar a distribuição dos óbitos por câncer de colo do útero no Brasil.

\section{MÉTODOS}

Trata-se de um estudo descritivo e ecológico, com uma abordagem quantitativa. O estudo foi desenvolvido por meio de dados secundários, do período de 2010 a 2019, sendo utilizado o Sistema de Informações sobre Mortalidade (SIM) para obtenção dos óbitos por câncer de colo do útero, e o Instituto Brasileiro de Geografia e Estatística (IBGE) para a obtenção das estimativas populacionais.

O estudo considerou a população feminina, e a área de abrangência do estudo foi todo o território nacional, que apresenta uma população estimada em, aproximadamente, 210 milhões de habitantes ${ }^{9}$. O estudo construiu o indicador da Taxa de Mortalidade por Câncer de Colo do Útero, a qual é calculada pela divisão da quantidade de óbitos pela população feminina residente, sendo multiplicados por 100 mil. Além disso, também foi realizada a distribuição geográfica das médias das taxas por meio de um mapa temático.

Em relação às variáveis, o estudo considerou as seguintes: ano do óbito (no período de 2010 a 2019); localização geográfica (Unidades Federativas); faixa etária (0 a 9 anos, 10 a 19 anos, 20 a 39 anos, 40 a 59 anos, 60 e mais); raça/cor (Branca, Negra, Amarela e Indígena); e escolaridade (nenhuma, 1 a 3 anos, 4 a 7 anos, 8 a 11 anos e 12 anos e mais).

A coleta dos dados foi realizada por meio dos Sistemas de Informação em Saúde, disponíveis no Departamento de Informática do SUS (DATASUS). No SIM, foram coletados os dados de óbitos por câncer de colo do útero, sendo considerado o Capítulo II (Neoplasias) e a Categoria C53 (Neoplasia maligna do colo do útero) da Classificação Estatística Internacional de Doenças e Problemas Relacionados à Saúde (CID-10). Utilizouse o IBGE para a coleta das estimativas populacionais dos residentes do Brasil.
As Taxas de Mortalidade por Câncer de Colo do Útero foram calculadas segundo as Unidades Federativas, sendo consideradas as médias do período analisado para a construção do mapa. 0 processamento e a análise dos dados foram realizados por meio do editor de planilha Microsoft Office Excel e software SIG QGIS 3.16, sendo apresentados em tabelas, gráfico e mapa temático, por meio das frequências absolutas e relativas.

Em relação aos aspectos éticos, o presente estudo foi desenvolvido por meio de dados secundários, de domínio público, garantindo a confidencialidade das informações pessoais dos envolvidos. Desse modo, de acordo com a Resolução do Conselho Nacional de Saúde $n^{\circ} 466$, está dispensada a submissão ao Comitê de Ética e Pesquisa, uma vez que o estudo garante a proteção das pesquisas com seres humanos ${ }^{10}$.

\section{RESULTADOS}

O Brasil apresentou uma tendência crescente do número de óbitos, sendo 4.986 óbitos no início da série histórica (2010) e atingindo 6.596 óbitos ao final (2019). Esse resultado também é observado na Taxa de Mortalidade por Câncer de Colo do Útero, em que o último ano da série (2019) apresentou a maior taxa de toda a série, ocorrendo 6,14 óbitos a cada 100 mil mulheres (figura 1).

Figura 1. Número de óbitos e Taxa de Mortalidade por Câncer de Colo do Útero segundo ano. Brasil, 2010 a 2019.

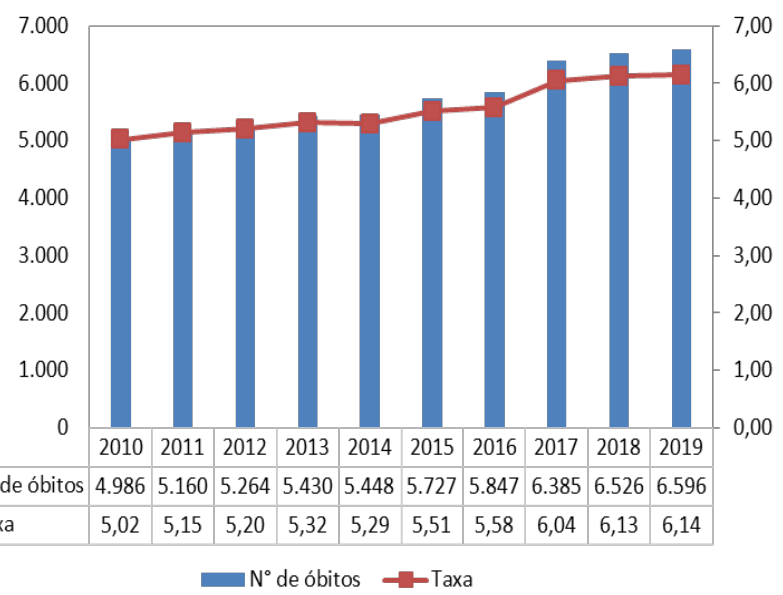

Fonte: Sistema de Informações sobre Mortalidade (SIM), 2010 a 2019; Instituto Brasileiro de Geografia e Estatística (IBGE), 2010 a 2019.

Em relação à faixa etária, o número e a proporção de óbitos por câncer de colo do útero são apresentados na tabela 1. No período analisado, destaca-se a faixa etária de 60 anos e mais, com uma maior incidência, apresentando um total de 24.364, o equivalente a $42,47 \%$ dos óbitos. Considerando outras faixas etárias, entre as mulheres de 40 a 59 anos, ocorreu um total de $24.095(42,00 \%)$ de óbitos, de 20 a 39 anos ocorreram 8.871 $(15,46 \%)$ e, em adolescentes de 10 a 19 anos, foram registrados $36(0,06 \%)$ óbitos. 
3 Óbitos por câncer de colo do útero no Brasil

Tabela 1. Número e proporção de óbitos por Câncer de Colo do Útero segundo Faixa Etária e ano. Brasil, 2010 a 2019.

\begin{tabular}{|c|c|c|c|c|c|c|c|c|c|c|c|}
\hline \multirow{2}{*}{ Ano } & \multicolumn{2}{|c|}{10 a 19 anos } & \multicolumn{2}{|c|}{20 a 39 anos } & \multicolumn{2}{|c|}{40 a 59 anos } & \multicolumn{2}{|c|}{60 e mais } & \multicolumn{2}{|c|}{ Ignorado } & \multirow[b]{2}{*}{ Total } \\
\hline & $\mathbf{N}$ & $\%$ & $\mathbf{N}$ & $\%$ & $\mathbf{N}$ & $\%$ & $\mathbf{N}$ & $\%$ & $\mathbf{N}$ & $\%$ & \\
\hline 2010 & 1 & 0,02 & 732 & 14,68 & 2.194 & 44,00 & 2.059 & 41,30 & 0 & 0,00 & 4.986 \\
\hline 2011 & 3 & 0,06 & 740 & 14,34 & 2.232 & 43,26 & 2.185 & 42,34 & 0 & 0,00 & 5.160 \\
\hline 2012 & 5 & 0,09 & 792 & 15,05 & 2.241 & 42,57 & 2.225 & 42,27 & 1 & 0,02 & 5.264 \\
\hline 2013 & 5 & 0,09 & 795 & 14,64 & 2.329 & 42,89 & 2.301 & 42,38 & 0 & 0,00 & 5.430 \\
\hline 2014 & 5 & 0,09 & 856 & 15,71 & 2.245 & 41,21 & 2.342 & 42,99 & 0 & 0,00 & 5.448 \\
\hline 2015 & 1 & 0,02 & 912 & 15,92 & 2.380 & 41,56 & 2.433 & 42,48 & 1 & 0,02 & 5.727 \\
\hline 2016 & 5 & 0,09 & 930 & 15,91 & 2.493 & 42,64 & 2.418 & 41,35 & 1 & 0,02 & 5.847 \\
\hline 2017 & 7 & 0,11 & 1.024 & 16,04 & 2.637 & 41,30 & 2.717 & 42,55 & 0 & 0,00 & 6.385 \\
\hline 2018 & 3 & 0,05 & 1.063 & 16,29 & 2.635 & 40,38 & 2.825 & 43,29 & 0 & 0,00 & 6.526 \\
\hline 2019 & 1 & 0,02 & 1.027 & 15,57 & 2.709 & 41,07 & 2.859 & 43,34 & 0 & 0,00 & 6.596 \\
\hline Total & 36 & 0,06 & 8.871 & 15,46 & 24.095 & 42,00 & 24.364 & 42,47 & 3 & 0,01 & 57.369 \\
\hline
\end{tabular}

Fonte: Sistema de Informações sobre Mortalidade (SIM), 2010 a 2019.

O número e a proporção de câncer de colo do útero segundo raça/cor são apresentados na tabela 2 . No total de óbitos verificados, durante a série histórica, a mortalidade foi maior na população negra, com um total de 30.786 óbitos, o equivalente a $53,66 \%$. Em relação às outras raças/cor, a população branca apresentou $23.850(41,57 \%)$, a população amarela $247(0,43 \%)$ e a população indígena 294 (0,51\%) óbitos.
Quando comparado o primeiro com o último ano da série histórica, nota-se uma redução na proporção de óbitos na população branca, passando de 44,91\% para 40,01\%. Enquanto isso, observa-se um aumento na proporção da polução negra, sendo $48,94 \%$ no ano de 2010 e $53,66 \%$ no ano de 2019. Destaca-se, também, um total de 2.192 (3,82\%) óbitos, em que a informação referente à raça/cor foi ignorada.

Tabela 2. Número e proporção de óbitos por Câncer de Colo do Útero segundo Raça/Cor e ano. Brasil, 2010 a 2019.

\begin{tabular}{|c|c|c|c|c|c|c|c|c|c|c|c|}
\hline \multirow{2}{*}{ Ano } & \multicolumn{3}{|c|}{ Branca } & Negra & \multicolumn{2}{|c|}{ Amarela } & \multicolumn{2}{|c|}{ Indígena } & \multicolumn{3}{|c|}{ Ignorado } \\
\hline & $\mathbf{N}$ & $\%$ & $\mathbf{N}$ & $\%$ & $\mathbf{N}$ & $\%$ & $\mathbf{N}$ & $\%$ & $\mathbf{N}$ & $\%$ & Total \\
\hline 2010 & 2.239 & 44,91 & 2.440 & 48,94 & 25 & 0,50 & 20 & 0,40 & 262 & 5,25 & 4.986 \\
\hline 2011 & 2.198 & 42,60 & 2.624 & 50,85 & 26 & 0,50 & 17 & 0,33 & 295 & 5,72 & 5.160 \\
\hline 2012 & 2.260 & 42,93 & 2.748 & 52,20 & 17 & 0,32 & 22 & 0,42 & 217 & 4,12 & 5.264 \\
\hline 2013 & 2.254 & 41,51 & 2.898 & 53,37 & 27 & 0,50 & 26 & 0,48 & 225 & 4,14 & 5.430 \\
\hline 2014 & 2.242 & 41,15 & 2.935 & 53,87 & 15 & 0,28 & 23 & 0,42 & 233 & 4,28 & 5.448 \\
\hline 2015 & 2.397 & 41,85 & 3.075 & 53,69 & 19 & 0,33 & 32 & 0,56 & 204 & 3,56 & 5.727 \\
\hline 2016 & 2.366 & 40,47 & 3.222 & 55,11 & 32 & 0,55 & 27 & 0,46 & 200 & 3,42 & 5.847 \\
\hline 2017 & 2.605 & 40,80 & 3.496 & 54,75 & 31 & 0,49 & 48 & 0,75 & 205 & 3,21 & 6.385 \\
\hline 2018 & 2.650 & 40,61 & 3.631 & 55,64 & 27 & 0,41 & 39 & 0,60 & 179 & 2,74 & 6.526 \\
\hline 2019 & 2.639 & 40,01 & 3.717 & 56,35 & 28 & 0,42 & 40 & 0,61 & 172 & 2,61 & 6.596 \\
\hline Total & 23.850 & 41,57 & 30.786 & 53,66 & 247 & 0,43 & 294 & 0,51 & 2.192 & 3,82 & 57.369 \\
\hline
\end{tabular}

Fonte: Sistema de Informações sobre Mortalidade (SIM), 2010 a 2019.

Em relação ao nível de escolaridade, os dados são apresentados na tabela 3. No total de óbitos, durante a série histórica, a mortalidade de câncer de colo do útero entre as mulheres que não apresentam nenhuma escolaridade foi de 9.451, o equivalente ao proporcional de $16,47 \%$ dos óbitos. Em relação aos outros níveis de escolaridade, destaca-se a população feminina que apresentou 1 a 3 anos e 4 a 7 anos, com um total de $13.590(23,69 \%)$ e $12.585(21,94 \%)$, respectivamente.
As mulheres com 8 a 11 anos apresentaram um total de 9.197 $(16,03 \%)$, enquanto o nível de escolaridade de 12 anos e mais apresentou apenas 2.481 (4,32\%). Em relação ao campo ignorado, nota-se uma redução na proporção, passando de $22,52 \%$ no início da série histórica para $14,27 \%$ ao final. No entanto, essa proporção ainda é muita alta, apresentando um total de 10.065 (17,54\%) óbitos sem a informação da escolaridade. 
Tabela 3. Número e proporção de óbitos por Câncer de Colo do Útero segundo Escolaridade e ano. Brasil, 2010 a 2019.

\begin{tabular}{|c|c|c|c|c|c|c|c|c|c|c|c|c|c|}
\hline \multirow{2}{*}{ Ano } & \multicolumn{2}{|c|}{ Nenhuma } & \multicolumn{2}{|c|}{1 a 3 anos } & \multicolumn{2}{|c|}{4 a 7 anos } & \multicolumn{2}{|c|}{8 a 11 anos } & \multicolumn{2}{|c|}{12 anos e mais } & \multicolumn{3}{|c|}{ Ignorado } \\
\hline & $\mathbf{N}$ & $\%$ & $\mathbf{N}$ & $\%$ & $\mathbf{N}$ & $\%$ & $\mathbf{N}$ & $\%$ & $\mathbf{N}$ & $\%$ & $\mathbf{N}$ & $\%$ & Total \\
\hline 2010 & 845 & 16,95 & 996 & 19,98 & 1.249 & 25,05 & 571 & 11,45 & 202 & 4,05 & 1.123 & 22,52 & 4.986 \\
\hline 2011 & 937 & 18,16 & 1.381 & 26,76 & 1.025 & 19,86 & 614 & 11,90 & 152 & 2,95 & 1.051 & 20,37 & 5.160 \\
\hline 2012 & 949 & 18,03 & 1.409 & 26,77 & 1.105 & 20,99 & 666 & 12,65 & 189 & 3,59 & 946 & 17,97 & 5.264 \\
\hline 2013 & 925 & 17,03 & 1.408 & 25,93 & 1.099 & 20,24 & 764 & 14,07 & 195 & 3,59 & 1.039 & 19,13 & 5.430 \\
\hline 2014 & 973 & 17,86 & 1.325 & 24,32 & 1.117 & 20,50 & 847 & 15,55 & 208 & 3,82 & 978 & 17,95 & 5.448 \\
\hline 2015 & 914 & 15,96 & 1.455 & 25,41 & 1.209 & 21,11 & 951 & 16,61 & 231 & 4,03 & 967 & 16,88 & 5.727 \\
\hline 2016 & 904 & 15,46 & 1.438 & 24,59 & 1.317 & 22,52 & 1.005 & 17,19 & 236 & 4,04 & 947 & 16,20 & 5.847 \\
\hline 2017 & 981 & 15,36 & 1.413 & 22,13 & 1.463 & 22,91 & 1.125 & 17,62 & 332 & 5,20 & 1.071 & 16,77 & 6.385 \\
\hline 2018 & 1.024 & 15,69 & 1.367 & 20,95 & 1.497 & 22,94 & 1.278 & 19,58 & 358 & 5,49 & 1.002 & 15,35 & 6.526 \\
\hline 2019 & 999 & 15,15 & 1.398 & 21,19 & 1.504 & 22,80 & 1.376 & 20,86 & 378 & 5,73 & 941 & 14,27 & 6.596 \\
\hline Total & 9.451 & 16,47 & 13.590 & 23,69 & 12.585 & 21,94 & 9.197 & 16,03 & 2.481 & 4,32 & 10.065 & 17,54 & 57.369 \\
\hline
\end{tabular}

Fonte: Sistema de Informações sobre Mortalidade (SIM), 2010 a 2019.

Além disso, o presente estudo analisou a distribuição geográfica dos óbitos por câncer de colo do útero no Brasil. Ao realizar uma análise regional, destaca-se a Região Norte, onde a maioria dos estados apresentou altas taxas de mortalidade, seguida pela Região Nordeste. Além disso, destacam-se as regiões do Sul e Sudeste, onde todos os estados apresentaram taxas médias entre 3,8 a 6,3 óbitos a cada 100 mil mulheres. Ao analisar de forma intrarregional, destacam-se os estados do Amazonas e Maranhão, pertencentes às regiões do Norte e Nordeste, respectivamente (figura 2).

Figura 2. Distribuição geográfica da Taxa Média da Mortalidade por Câncer de Colo do Útero segundo Unidade Federativa. Brasil, 2010 a 2019.

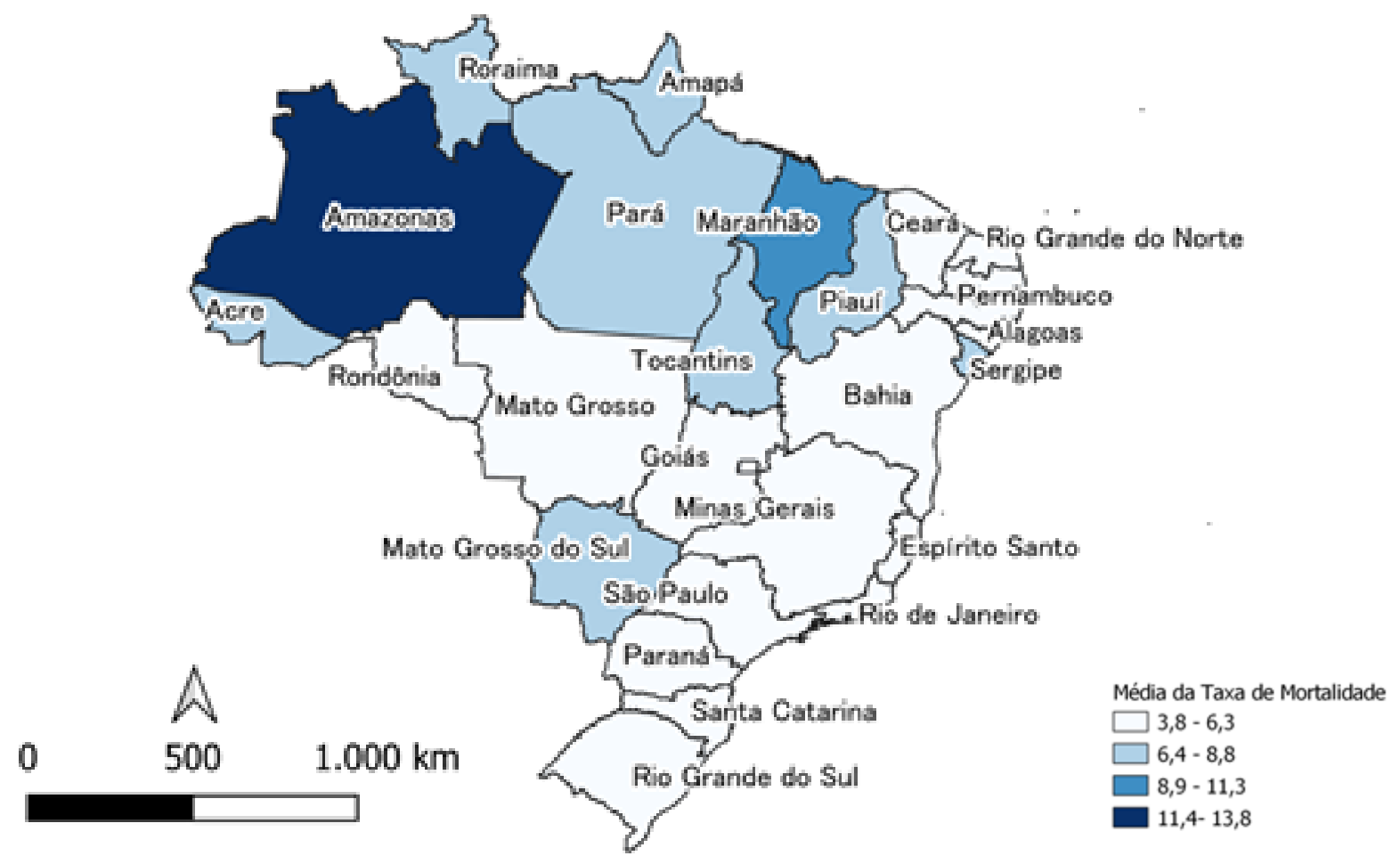

Fonte: Sistema de Informações sobre Mortalidade (SIM), 2010 a 2019; Instituto Brasileiro de Geografia e Estatística (IBGE), 2010 a 2019. 


\section{DISCUSSÃO}

O câncer de colo do útero é uma patologia que se manifesta de forma silenciosa e assintomática, sendo capaz de acometer órgãos próximos, demonstrando, dessa forma, a magnitude da doença. A detecção precoce e o tratamento em tempo oportuno são estratégias fundamentais para a redução da mortalidade pela neoplasia, sendo o exame citopatológico a ferramenta chave para o diagnóstico, podendo oferecer uma melhor qualidade de vida quando detectado precocemente $\mathrm{p}^{11,12}$.

O presente estudo indicou uma tendência crescente no número de óbitos por câncer de colo do útero no Brasil. Em um estudo realizado no ano de 2020, o autor indica que a predisposição crescente no número de óbitos pela neoplasia e no índice de mortalidade são resultados esperados no país, visto que é uma doença recorrente em países que estão em desenvolvimento. Fatores como baixa renda, baixo nível de escolaridade e dificuldade ao acesso aos serviços de saúde são indicados como determinantes para o aumento dos óbitos por câncer de colo do útero ${ }^{13}$.

Tallon e outros colaboradores (2020) realizaram um estudo referente às tendências de mortalidade por câncer de colo do útero, para os anos de 2012 a 2016, em todo o território nacional. Os achados do estudo indicaram um aumento no número de óbitos pela neoplasia, em que, no primeiro ano do período analisado, foram registrados 5.264 óbitos, aumentando para 5.847 no último ano. Dessa forma, os achados se assemelham com os identificados no presente estudo ${ }^{14}$.

Além disso, no presente estudo, destaca-se, também, a faixa etária de 60 anos e mais, com uma proporção de 42,47\% dos óbitos (tabela 1). As mulheres acima de 60 anos não são contempladas no programa de rastreio, desde modo, as lesões intraepiteliais não são detectadas de forma precoce nessa população ${ }^{15}$. Esse resultado pode ser correlacionado com os níveis de escolaridade, em que a população idosa apresenta altos índices de analfabetismo quando comparado a mulheres adultas; dessa forma, pode ser classificado como um mau prognóstico, tornando-se um fator de risco expressivo ${ }^{16}$.

Segundo Selva e outros autores (2020), o grupo das neoplasias é uma das causas mais predominantes dos óbitos na população idosa. Nos resultados, os autores identificaram um aumento no número de casos de câncer de colo do útero entre essa faixa etária, em que $70 \%$ dos óbitos pertenciam a esta população16. De acordo com Verzaro \& Sardinha (2018), as mulheres acima de 64 anos, diagnosticadas com a neoplasia, apresentavam HPV. Além disso, essas mulheres mantêm-se mais tempo retardando os sintomas da doença, não procurando atendimento médico ginecológico. De outro modo, as mulheres jovens buscam o atendimento em tempo oportuno ${ }^{17}$. Dessa forma, os achados do estudo indicam a necessidade de reformular as diretrizes do rastreamento do câncer de colo do útero.

Segundo Souza (2020), no período de 2002 a 2017, foram notificados 101.043 óbitos por câncer de colo do útero, sendo 49.176 (48,7\%) óbitos em mulheres brancas e 50.919 (50,39\%) óbitos em mulheres negras. Desse modo, colabora com os achados do presente estudo. Além disso, ao analisar artigos sobre raça/cor relacionados à mortalidade por câncer de colo do útero no Brasil, o autor indicou que a maioria dos estudos realizados sobre a temática no país não considera a variável raça/cor ${ }^{18}$.

Em relação a um estudo, Oliveira, Oliveira \& Nogueira (2019) apontaram que as mulheres que estudaram em um intervalo de 12 anos e mais apresentaram uma baixa taxa de mortalidade; dessa forma, corroborando com os achados do presente estudo. Dessa maneira, o nível de escolaridade tem interferência com as medidas de prevenção, em que o baixo nível de escolaridade dificulta o processo de informação à saúde. No entanto, este resultado indica a necessidade de os serviços e profissionais de saúde desenvolverem estratégias de educação em saúde específicas para essa população ${ }^{19}$.

De acordo com Tallon e outros colaboradores (2020), a região Norte apresentou os maiores índices de óbitos por câncer de colo do útero, em seguida, a região Nordeste; colaborando, dessa maneira, com os achados do presente estudo. Os autores também destacaram a região Centro-Oeste, que vem apresentando um aumento na mortalidade pela neoplasia. Além disso, apontaram a existência de divergências em relação ao acesso aos serviços de saúde entre as regiões do país, indicando que as condições socioeconômicas ainda se caracterizam como um obstáculo para o sistema de saúde brasileiro ${ }^{14}$.

Dessa maneira, é importante salientar e construir o perfil das pacientes com câncer de colo do útero. No Brasil, as baixas condições socioeconômicas, como nível de escolaridade e renda, ainda se caracterizam como um fator de risco para a ocorrência do óbito pela neoplasia e para o acesso aos serviços de saúde. Dessa forma, analisar o perfil é essencial para identificar possíveis grupos vulneráveis e reorientar as ações e os serviços de saúde para a população feminina ${ }^{20}$.

\section{CONCLUSÕES}

O Brasil apresentou uma tendência crescente para a mortalidade por câncer de colo do útero; no entanto, a distribuição geográfica dos óbitos não é uniforme em todo o território nacional, indicando a persistência das desigualdades regionais no país. Além disso, as elevadas proporções de mortalidade na população negra e nas mulheres com baixos níveis de escolaridade apontam uma possível relação entre a ocorrência dos óbitos e as condições demográficas e socioeconômicas da população feminina.

Dessa forma, a análise do perfil dos óbitos por câncer de colo do útero é fundamental para a identificação dos grupos populacionais mais vulneráveis, contribuindo para o 
direcionamento das ações e dos serviços de saúde. Além disso, esta análise é essencial para o processo de planejamento em saúde e para a elaboração e o aprimoramento das políticas públicas de saúde voltadas para a prevenção do câncer de colo do útero.

\section{REFERÊNCIAS}

1. Vaz GP, Bitencourt EL, Martins GS, Carvalho AABD, Reis PM Júnior. Perfil Epidemiológico do Câncer de Colo do útero na Região Norte do Brasil no Período de 2010 a 2018. Rev Patol Tocantins [Internet]. 2020 Jul [acesso 2021 Jun 26]; 7(2):114-117. Disponível em: https://sistemas.uft.edu.br/periodicos/index. php/patologia/article/view/8750. doi: https://doi.org/10.20873/uft.24466492.2020v7n2p114.

2. Ceolin R, Nasi C, Paz AA, Lich GFC. Perfil de mortalidade por câncer de colo do útero no período de 2005-2014. Rev Enferm Centro-Oeste Mineiro [Internet]. 2020 Abr-Jun [acesso 2021 Jun 26]; 20(2): 643-651. Disponivel em: http:// www.seer.ufsj.edu.br/index.php/recom/article/view/1806. doi: https://doi. org/10.19175/recom.v8i0.1806.

3. Damacena AM, Luz LL, Mattos IE. Rastreamento do câncer do colo útero em Teresina, Piauí estudo avaliativo dos dados do sistema de informação do câncer do clo do útero, 2006-2013. Epidemiol. Serv. Saude [Internet]. 2017 JanMar [acesso 2021 Jun 27]; 26(1): 71-80. Disponível em: https://www.scielosp. org/article/ress/2017.v26n1/71-80/. doi: https://doi.org/10.5123/S167949742017000100008.

4. Almeida CMC, Souza AN, Bezerra RS, Lima FLO, Izabel TDSS. Principais fatores de risco associados ao desenvolvimento do câncer de colo do útero, com ênfase para o Papilomavírus humano (HPV): um estudo de revisão. Res Soc Devel [Internet]. 2021 Jan [acesso 2021 Jun 28]; 10(1): 1-11. Disponível em: https://rsdjournal.org/index.php/rsd/article/view/11634. DOI: http://dx.doi. org/10.33448/rsd-v10i1.11634.

5. Holanda JCR, Araújo MHHPO, Nascimento WG, Gama MPA, Souza CSM. Uso do Protocolo de saúde da mulher na prevenção do câncer de colo do útero. Rev baiana enferm [Internet]. 2021 [acesso 2021 Jun 28]; 35: e39014. Disponível em: https://periodicos.ufba.br/index.php/enfermagem/article/view/39014/24557. doi: http://dx.doi.org/10.18471/rbe.v35.39014.

6. George TJ. Factors influencing utilization of cervical cancer screening services among women - A cross sectional survey. Clin Epidemiol Glob Health [Internet]. 2021 Jul-Sep [acesso 2021 Jun 29]; 11: 2213-3984. Disponível em: https://www. sciencedirect.com/science/article/pii/S2213398421000567?via\%3Dihub. doi: https://doi.org/10.1016/j.cegh.2021.100752.

7. Lopes VAS, Ribeiro JM. Fatores limitadores e facilitadores para o controle do câncer de colo do útero: uma revisão de literatura. Ciênc saúde coletiva [Internet]. 2019 Set. [acesso 2021 Jun 30]; 24(9): 3431-3442. Disponível em: https://www.scielosp.org/article/csc/2019.v24n9/3431-3442/pt/. doi: https:// doi.org/10.1590 /1413-81232018249.32592017.

8. Silva EGDS, Silva RKDS, Oliveira LDL, Leal BMN, Carvalho MDL, Silva BGS, et al. Letramento em saúde e prevenção do câncer do colo de útero. Braz. J Develop [Internet]. 2020 Jul [acesso 2021 Jul 1]; 6(7): 43439-43448. Disponível em: https://www.brazilianjournals.com/index.php/BRJD/article/ view/12666/10632. doi: https://doi.org/10.34117/bjdv6n7-093.

9. Instituto Brasileiro de Geografia e Estatística. Projeção da população do Brasil e das Unidades da Federação [Internet]. Rio de Janeiro: IBGE; 2021 [acesso 2021 Jul 1]. Disponível em: https://www.ibge.gov.br/apps/populacao/ projecao/index.html.

10. Brasil. Resolução no 466, de 12 de dezembro de 2012. Aprova diretrizes e normas regulamentadoras de pesquisa envolvendo seres humanos [Internet]. Diário Oficial da União. 2012 Dez [acesso 2021 Jul 5]. Disponível em: https:// bvsms.saude.gov.br/bvs/saudelegis/cns/2013/res0466_12_12_2012.html.

11. Aoyama EDA, Pimentel ADS, Andrade JSD, Daniel WV, Souza RAGD, Lemos LR. Assistência de enfermagem na prevenção do câncer de colo do útero. Braz J Health Rev [Internet]. 2018 Dez [acesso 2021 Jul 8]; 162-170. Disponível em: https://www.brazilianjournals.com/index.php/BJHR/article/view/877.

12. Maciel LMA, Souza RAG, Aoyama EDA. A importância do exame Papanicolau realizado pelo enfermeiro para o diagnóstico do câncer no colo uterino. Rev Bras Inter Saúde [Internet]. 2020 Jan [acesso 2021 Jul 8]; 2(2): 88-92. Disponível em: https://revistarebis.rebis.com.br/index.php/rebis/article/view/95/88.

13. Vasconcelos KST. Relação das condições socioeconômicas com o número de óbitos por câncer de colo de útero [trabalho de conclusão de curso]. Campinas (SP): Ariquemes: Faculdade de Educação e Meio Ambiente; 2020.

14. Tallon B, Monteiro D, Soares L, Rodrigues N, Morgado F. Tendências da mortalidade por câncer de colo no Brasil em 5 anos (2012-2016). Saúde debate [Internet]. 2020 Abr-Jun [acesso 2021 Jul 6]; 44(125): 362-371. Disponível em: https://www.scielo.br/j/sdeb/a/rtpBHcDBNzw45zrxFNkw3sf/?lang=pt. doi: https://doi.org/ 10.1590/0103-1104202012506.

15. Silva RCGD, Silva ACDO, Peres AL, Oliveira SRD. Perfil de mulheres com câncer de colo do útero atendidas para tratamento em centro de oncologia. Rev. Bras. Saúde Mater. Infant. [Internet]. 2018 Out-Dez [acesso 2021 Jul 7]; 18(4): 703-710. Disponível em: https://www.scielo.br/j/rbsmi/a/ PgGpzdL6HqQTsk8RPLVD9JR/?lang=pt. doi: https://doi.org/10.1590/180693042018000400002

16. Selva ACV, Guaraná CVPDS, Dias VDS, Sales JT, Azevedo CRAS. Estudo de coorte prospectivo de pacientes com câncer de colo do útero: a idade é um fator determinante?. Braz J Health Rev [Internet]. 2020 Jul [acesso 2021 Jul 8]; 3(4): 8679-8695. Disponível em: https://www.brazilianjournals.com/index.php/ BJHR/article/view/13491. doi: https://doi.org/10.34119/bjhrv3n4-115.

17. Verzaro PM, Sardinha AHDL. Caracterização sociodemográfica e clínica de idosas com câncer do colo do útero. Rev. Salud Pública [Internet]. 2018 Out [acesso $2021 \mathrm{Jul}$ 10]; 20(6): 718-724. Disponível em: https://www.scielosp. org/article/rsap/2018.v20n6/718-724/. doi: https://doi.org/10.15446/rsap. V20n6.69297.

18. Souza ASPD. Diferenças raciais na mortalidade por câncer de colo do útero no Brasil. [dissertação]. São Paulo (SP): Universidade de São Paulo; 2019.

19. OLIVEIRA LLP, Oliveira HCC, Nogueira EC. Taxa de mortalidade por câncer de colo de útero em Aracaju - SE no período de 2014-2016. In: Anais do 20 Congresso Internacional de Enfermagem; 2019 Maio 6-10; Aracaju: Universidade Tiradentes; 2019.

20. Silva MDT, Marques RB, Costa LO. Câncer de colo do útero: barreiras preventivas no século 21. Cad Medicina. [Internet]. 2021 Abr [acesso 2021 Jul 14]; 3(1): 7610-7626. Disponível em: http://www.revista.unifeso.edu.br/ index.php/ cadernosdemedicinaunifeso/article/view/2252. doi: 10.34119/ bjhrv4n2-300.

\section{Como citar este artigo/How to cite this article:}

Fonseca TAA, Silva DTA, Silva MTA. Distribuição dos óbitos por câncer de colo do útero no Brasil. J Health Biol Sci. 2021; 9(1):1-6. 